\section{Peso y talla en niños con retinoblastoma}

\section{RESUMEN}

Introducción: la talla baja se ha descrito como característica clínica de niños con retinoblastoma. Esta particularidad puede estar relacionada directamente con la enfermedad de base o con factores externos.

Objetivo: el propósito de esta investigación fue conocer la frecuencia de talla y peso bajos en niños mexicanos con retinoblastoma y correlacionar los valores antropométricos con variables de la enfermedad y ambientales.

Materiales y métodos: se analizaron expedientes clínicos de 346 pacientes con retinoblastoma. Se obtuvieron los datos de peso y talla referidos al ingreso; antes del tratamiento. Se comparó el valor de Z con las referencias de la distribución normal de la población mexicana. La asociación de las medidas antropométricas con las variables clínicas, sociales y genéticas se estableció con la prueba de $\chi^{2}$.

Resultados: la talla y el peso promedio de los niños con retinoblastoma fueron significativamente más bajos que los de la población general ( $p<0.001$ y $p=0.013$, respectivamente). La talla y peso bajos fueron más frecuentes en niños con enfermedad avanzada, provenientes de medio rural y de bajo nivel socioeconómico.

Conclusiones: los déficits en peso y talla en niños con retinoblastoma se deben a factores relacionados con enfermedad avanzada al momento del diagnóstico, lo cual a su vez es consecuencia de la situación de marginación de la población rural mexicana. Dado que la mayoría de las variables sociales y ambientales están muy relacionadas y que no podemos descartar posibles factores genéticos, las conclusiones de este estudio deben verificarse analizando las medidas de los padres y hermanos y establecer grupos comparativos para controlar las variables confusas.

Palabras clave: retinoblastoma, talla baja, peso bajo.
Cecilia Ridaura-Sanz ${ }^{1}$

Chiharu Murata

Fátima Ysusi-Mendoza ${ }^{3}$

Carlos Leal-Leal ${ }^{4}$

${ }^{1}$ Departamento de Patología del Instituto Nacional de Pediatría.

${ }^{2}$ Subdirección de Investigación Médica del Instituto Nacional de Pediatría Departamento de Metodología de la Investigación.

${ }^{3}$ Pediatra. Oncología Pediátrica del Instituto Nacional de Pediatría.

${ }^{4}$ Oncólogo. Pediatra Responsable de la clínica de Retinoblastoma, Oncología Pediátrica del Instituto Nacional de Pediatría.
Recibido: 23 de septiembre del 2014

Aceptado: 6 de febrero del 2015

Correspondencia: Dra. Cecilia Ridaura Sanz Departamento de Patología Instituto Nacional de Pediatría Teléfono 10845515

cridaura@gmail.com

Este artículo debe citarse como Ridaura-Sanz C, Murata C, Ysusi-Mendoza F, Leal-Leal C. Peso y talla en niños con retinoblastoma. Acta Pediatr Mex 2015;36:81-88. 
Methods: There were 346 patients with a histopathological diagnosis of retinoblastoma reported by the Oncology Service of the Instituto Nacional de Padiátría (INP). Weight and height were recorded in the patient charts prior to treatment. The Z-score was used to compare the study for mean somatometric value distribution with that of the data from the National Center for Health Statistics [NCHS]/Center for Disease Control and Prevention [CDC] and reference for Mexican population. The association of the variables related to the clinical, social and genetic aspects of retinoblastoma with the low weight and short height was assessed by the ratio chi square test.

Results: The average weight and the average height of the retinoblastoma population were significantly lower than those of the general population $(p<0.001$ and $p=0.013$, respectively). Low weight and Short stature in retinoblastoma patients was more frequent among those with advanced disease, rural environment and lower socioeconomic levels.

Conclusions: The weight and height deficit present in a large proportion of children with retinoblastoma is due to factors such as an advanced stage of the condition at diagnosis, which, in turn, is symptomatic in the rural populations of the poorest areas of Mexico. Given that most social and environmental variables are highly interrelated, and the occurrence of possible genetic factors throughout Mexico cannot be discarded, the conclusions of the present study should be verified by analyzing the somatometric measurements of the parents and relatives of the patients, in order to establish comparison group comparison to and to assent these confounding variables.

Key words: Retinoblastoma, Low weight, Low height.

\section{INTRODUCCIÓN}

El retinoblastoma es la neoplasia maligna intraocular más frecuente en niños; es mucho más habitual en los países menos industrializados y en poblaciones económicamente pobres y en áreas rurales..$^{1-3}$ De acuerdo con el Surveillance, Epidemiology and End Results, del National Cancer Institute, en Estados Unidos el retinoblastoma ocupa el décimo lugar en la frecuencia de neoplasias malignas en la infancia, mientras que en México ocupa el segundo o tercer lugar; ${ }^{4-6}$ es más frecuente en los niños provenientes de zonas rurales de Chiapas que en los de la Ciudad de México.7,8
Existen dos formas de la enfermedad: la hereditaria y la esporádica. La primera es más rara, con una mutación en todas las células somáticas en la posición 14 del brazo largo del cromosoma 13, con un patrón de herencia autosómica dominante y penetración genética completa. Suele verse en niños pequeños y puede ser bilateral. ${ }^{9-11}$ La forma esporádica es unilateral y la mutación sólo se encuentra en las células neoplásicas. Afecta con mayor frecuencia a niños de países pobres y aunque la etiología no se conoce se ha postulado la posible influencia de factores genéticos, nutricionales y ambientales. ${ }^{1,9,12,13}$ 
Entre las manifestaciones clínicas de esta enfermedad se han referido peso y talla bajos. En algunos casos esta ha sido la causa de inicio del estudio, particularmente cuando se asocia con dismorfias faciales como parte del síndrome de deleción de la banda ql4 del cromosoma $13 . .^{14,15}$

La talla baja parece ser una manifestación clínica en algunos casos de retinoblastoma. ${ }^{1,14-16}$ Dado que en la etiopatogenia del retinoblastoma intervienen tanto factores genéticos como ambientales, es posible que alguno de estos interfiera con el crecimiento de los niños afectados en etapas tempranas de su desarrollo. La forma hereditaria del retinoblastoma podría asociarse con talla baja, lo cual indicaría posiblemente que la detención del crecimiento forma parte de un síndrome genético. Por otro lado, la mayor frecuencia de retinoblastoma esporádico en poblaciones procedentes de medio rural y de nivel socioeconómico bajo podría explicar el déficit en peso y talla como parte de las características de los niños de esas zonas.

\section{Objetivo}

El propósito de este estudio es investigar la frecuencia de talla y peso bajos en una población de niños mexicanos con retinoblastoma y relacionar las medidas antropométricas con características propias de la enfermedad así como variables relacionadas con el ambiente.

\section{MATERIAL Y MÉTODOS}

Estudio observacional, transversal y descriptivo. Se tomaron los expedientes de todos los pacientes con diagnóstico histopatológico de retinoblastoma en ojos enucleados, atendidos en el Servicio de Oncología del Instituto Nacional de Pediatría entre el 1 de enero de 1993 y el 31 de diciembre del 2002. Se excluyeron los casos con tratamiento previo. Se registraron los datos de peso y talla de la hoja de ingreso de los pacientes.
Otras variables registradas de los expedientes fueron:

1. Etapa clínica del tumor: estadios I, II, III y IV de acuerdo con la clasificación de Pratt.

2. Localización del tumor: bilateral o unilateral.

3. Antecedentes heredofamiliares: presente o ausente.

4. Nivel socioeconómico: bajo, medioalto, de la categoría establecida en la institución.

5. Lugar de procedencia por zonas de la república de acuerdo con la clasificación de la Encuesta Nacional de Nutrición 1999: Norte) Baja California Norte, Baja California Sur, Coahuila, Chihuahua, Durango, Nuevo León, Sonora y Tamaulipas. Distrito Federal. Centro) Aguascalientes, Colima, Guanajuato, Jalisco, Estado de México (no conurbada con el DF), Michoacán, Morelos, Nayarit, Querétaro, San Luis Potosí, Sinaloa. Sur) Campeche, Chiapas, Guerrero, Hidalgo, Oaxaca, Puebla, Quintana Roo, Tabasco, Tlaxcala, Veracruz, Yucatán.

6. Ambiente rural o urbano del lugar de procedencia.

Las variables somatométricas empleadas para el peso y la talla fueron:

a. Puntuación de Z, calculada con base en el patrón de referencia del National Center for Health Statistics (NCHS)/Centers for Disease Control and Prevention [CDC]) y

b. Variable dicotómica de peso bajo y talla baja $\left(<-2\right.$ DE de NCHS/CDC). ${ }^{17-20}$

También se exploraron las asociaciones de las variables somatométricas con las variables relacionadas a la patología de interés, al medio ambiente y las de la observación clínica. 
Análisis estadístico: la comparación de la media de la distribución de variables somatométricas con la de la población de referencia (NCHS/ CDC) se hizo por medio de la puntuación Z. Para el análisis de frecuencia se calcularon la probabilidad por la razón de verosimilitud y la prueba exacta de Fisher.

\section{RESULTADOS}

\section{Características generales de la población}

Se incluyeron 346 casos con retinoblastoma con edades desde un mes a ocho años de edad (mediana de 24 meses). El 75\% de los casos ingresó antes de cumplir tres años y 50\% de los casos en los primeros dos años de vida. La distribución por género fue similar. Más de la mitad de los casos llegaron al hospital en estadios avanzados de la enfermedad con tumor extraocular (etapa III) o con metástasis a distancia (etapa IV). El $40 \%$ proviene de los estados del sur de México y hubo muy pocos de los estados del norte. La distribución entre rural y urbano fue similar. La gran mayoría de los casos procedía de medio socioeconómico bajo de acuerdo con la clasificación de Trabajo Social del Instituto Nacional de Pediatría. ${ }^{21}$ Solamente en 21 casos (6.06\%) se documentó antecedente familiar de la misma enfermedad y en casi la tercera parte de los casos el tumor era bilateral. Con estos dos datos: bilateralidad y antecedente familiar, se puede calificar razonablemente el retinoblastoma como hereditario, lo que ocurrió en $31 \%$ de los casos (Cuadro 1).

\section{Talla y peso}

Hubo 60 casos de bajo peso $(<-2 z)(17.3 \%)$ y $61 \%$ de talla baja $(<-2 z)(17.6 \%)$. En 36 casos ambos parámetros fueron bajos; en 24 casos el peso fue bajo con talla normal y en 25 la talla fue baja con peso normal.
Cuadro 1. Distribución de los 346 casos: aspectos clínicos, sociales y genéticos

\begin{tabular}{llcc}
\hline \multicolumn{1}{c}{ Variables } & Categorías & $\begin{array}{c}\text { Número } \\
\text { de casos }\end{array}$ & Proporción \\
Género & Femenino & 163 & 0.47 \\
& Masculino & 183 & 0.53 \\
Etapa clínica & I & 43 & 0.12 \\
del tumor & II & 130 & 0.38 \\
& III & 91 & 0.26 \\
Zona de la & IV & 82 & 0.24 \\
república & Centro & 16 & 0.05 \\
& Distrito Federal & 151 & 0.44 \\
Ambiente & Sur & 149 & 0.11 \\
& Rural & 177 & 0.40 \\
Nivel & Urbano & 169 & 0.51 \\
socioeconó- & Bajo & 258 & 0.49 \\
mico & Medio-alto & 88 & 0.75 \\
Antecedente & Presente & 21 & 0.25 \\
heredofami- & Ausente & 325 & 0.94 \\
liar & & & \\
Lateralidad & Bilateral & 99 & 0.29 \\
& Unilateral & 247 & 0.71 \\
Genética & Hereditaria & 239 & 0.69 \\
& Esporádica & 107 & 0.31 \\
& & &
\end{tabular}

La Figura 1 muestra la distribución de peso y talla en puntuación Z basada en los valores de referencia NCHS/CDC. En ambas variables hay discrepancia con el modelo normal, aunque más acentuado para el peso por la presencia de valores extremos (tres casos de $<-6 D E$, y un caso de -10DE). La distribución de la puntuación Z para el peso y la talla se encuentran disminuidas y esa diferencia fue estadísticamente significativa.

En los Cuadros 2 y 3 se resume el resultado de la comparación de las proporciones de bajos peso y talla $(<-2 \mathrm{DE}$ de los valores de referencia NCHS/ CDC) de los pacientes con retinoblastoma y las de las muestras de la Encuesta Nacional de Nutrición de 1999, en cuatro zonas de la república por separado y en escala nacional. ${ }^{20}$ Hubo una mayor proporción de casos con peso bajo entre los niños menores de 5 años con retinoblastoma comparada con los de la encuesta de esa misma 
A) Peso en valor de Z

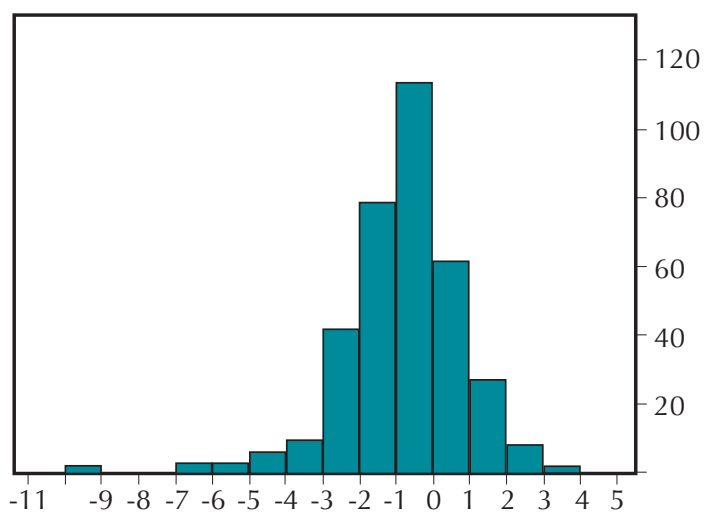

Media (DE): $-0.80(1.57$

IC $95 \%:-0.96,-0.63$

$\begin{array}{lcc}\text { Media } & -0.80 & -0.30 \\ \text { Desviación estándar } & 1.56 & 0.018 \\ \text { Sesgo } & -1.02 & \\ \text { Kurtosis } & 4.20 \\ \text { Prueba de ajuste a la normalidad } & \\ \text { (W de Shapiro-Wilk): } \mathrm{W}=0.958347, \mathrm{P}<0.0001\end{array}$

B) Talla en valor de Z

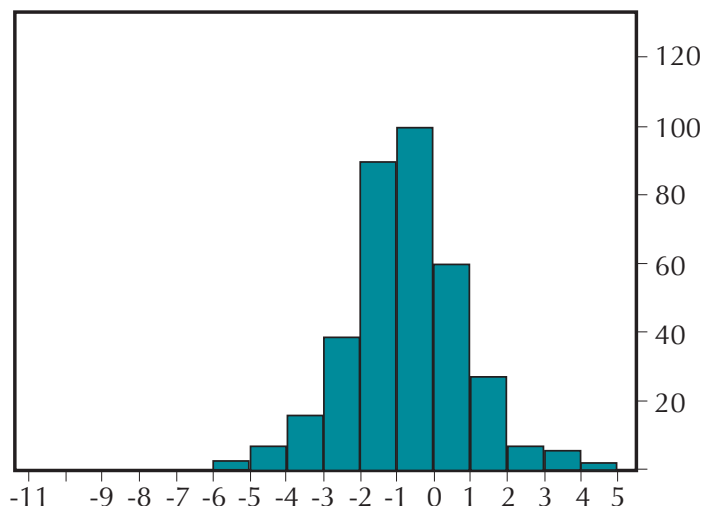

Media (DE): $-0.80(1.48)$

IC 95\%: $-0.96,-0.65$

Figura 1. Distribución de la puntuación Z de peso (A) y talla (B) de los 346 casos con retinoblastoma.

Cuadro 2. Proporción de peso bajo

\begin{tabular}{lcc}
\hline & \multicolumn{2}{c}{ 0 a 5 años de edad (N 328) } \\
& Retinoblastoma & ENN \\
Norte & $12.5(0,30.1)$ & $3.3(2.6,4.0)$ \\
Centro & $16.2(10.1,22.3)$ & $6.1(5.0,7.2)$ \\
Distrito Federal & $7.9(0,16.8)$ & $6.8(3.9,9.7)$ \\
Sur & $23.5(16.2,30.8)$ & $12.0(11.0,13.1)$ \\
Nacional & $18.0(13.8,22.2)$ & $7.6(6.9,8.3)$
\end{tabular}

Los valores en la columna de retinoblastoma son: estimación puntual de la proporción (límites inferior y superior de IC 95\%). ENN: Encuesta Nacional de Nutrición 1999.

edad (18 vs. 7.6\%). Esta diferencia estuvo presente en todas las zonas del país, a excepción del Distrito Federal donde la proporción de bajo peso de 7.9\% cae dentro de los límites de la población de la Encuesta Nacional de Nutrición (Cuadro 2). La proporción de talla baja (Cuadro 3) de los niños con retinoblastoma no mostró diferencia con la población nacional ni en la distribución por zonas.
Cuadro 3. Proporción de talla baja (N 328)

\begin{tabular}{lcc}
\hline & \multicolumn{2}{c}{$\mathbf{0}$ a $\mathbf{5}$ años de edad } \\
& Retinoblastoma & ENN \\
Norte & $6.3(0,19.1)$ & $7.1(5.9,8.3)$ \\
Centro & $16.9(10.7,23.1)$ & $14.5(12.4,16.6)$ \\
Distrito Federal & $7.9(0,16.8)$ & $13.1(9.6,16.7)$ \\
Sur & $22.7(15.5,29.9)$ & $29.2(27.0,31.5)$ \\
Nacional & $17.7(13.5,21.8)$ & $17.8(16.6,19.0)$
\end{tabular}

Los valores en la columna de retinoblastoma son: estimación puntual de la proporción (límites inferior y superior de IC 95\%). ENN: Encuesta Nacional de Nutrición 1999.

\section{Asociación de las variables de peso y talla con los aspectos clínicos, sociales y genéticos del retinoblastoma}

Los Cuadros 4 y 5 muestran la relación antropométrica con las variables independientes. Hay asociación entre peso y talla bajos para las variables de etapa clínica del tumor, procedencia 
Cuadro 4. Asociación de las variables que caracterizan los aspectos clínicos, sociales y genéticos con la variable peso bajo $(<-2 \mathrm{DE})$; esta última es variable dependiente

\begin{tabular}{|c|c|c|c|c|}
\hline Variables independientes & Categorías & $\begin{array}{l}\text { Número de casos de bajo peso y su } \\
\text { proporción }(\%) \text { en la categoría }\end{array}$ & $\chi^{2}$ & $P$ \\
\hline Género & Femenino Masculino & $\begin{array}{l}27 / 163(16.6) \\
33 / 183(18.0)\end{array}$ & 0.130 & 0.7186 \\
\hline Etapa clínica de tumoración & $\begin{array}{l}\text { I } \\
\text { II } \\
\text { III } \\
\text { IV }\end{array}$ & $\begin{array}{c}7 / 43(16.3) \\
15 / 130(11.5) \\
12 / 91(13.2) \\
26 / 82(31.7)\end{array}$ & 14.589 & 0.0022 \\
\hline Zonas de la república & $\begin{array}{l}\text { Norte } \\
\text { Centro } \\
\text { Distrito Federal } \\
\text { Sur }\end{array}$ & $\begin{array}{c}2 / 16(12.5) \\
24 / 151(15.9) \\
3 / 39(7.7) \\
31 / 140(22.1)\end{array}$ & 5.690 & 0.1277 \\
\hline Ambiente rural/urbano & $\begin{array}{l}\text { Rural } \\
\text { Urbano }\end{array}$ & $\begin{array}{c}45 / 177(25.4) \\
15 / 169(8.9)\end{array}$ & 17.206 & $<0.0001$ \\
\hline Nivel socieconómico & $\begin{array}{l}\text { Bajo } \\
\text { Medio-alto }\end{array}$ & $\begin{array}{l}54 / 258(20.9) \\
6 / 88(6.8)\end{array}$ & 10.655 & 0.0011 \\
\hline Genética del retinoblastoma & $\begin{array}{l}\text { Hereditario } \\
\text { Esporádico }\end{array}$ & $\begin{array}{l}18 / 107(16.8) \\
42 / 239(17.6)\end{array}$ & 0.029 & 0.8643 \\
\hline
\end{tabular}

* P en distribución de $\chi^{2}$ por la razón de verosimilitud.

Cuadro 5. Asociación de las variables que caracterizan los aspectos clínicos, sociales y genéticos con la variable talla baja $(<-2 \mathrm{DE})$; esta última es variable dependiente

\begin{tabular}{|c|c|c|c|c|}
\hline Variables independientes & Categorías & $\begin{array}{l}\text { Número de casos de talla baja y su } \\
\text { proporción }(\%) \text { en la categoría }\end{array}$ & $\chi^{2}$ & $\boldsymbol{P}$ \\
\hline Género & Femenino Masculino & $\begin{array}{l}25 / 163(15.3) \\
36 / 183(19.7)\end{array}$ & 1.122 & 0.2895 \\
\hline Etapa clínica de tumoración & $\begin{array}{l}\text { I } \\
\text { II } \\
\text { III } \\
\text { IV }\end{array}$ & $\begin{array}{c}6 / 43(14.0) \\
18 / 130(13.9) \\
12 / 91(13.2) \\
23 / 82(30.5)\end{array}$ & 11.158 & 0.0109 \\
\hline Zonas de la república & $\begin{array}{l}\text { Norte } \\
\text { Centro } \\
\text { Distrito Federal } \\
\text { Sur }\end{array}$ & $\begin{array}{c}1 / 16(6.3) \\
25 / 151(16.6) \\
3 / 39(7.7) \\
32 / 140(22.9)\end{array}$ & 7.612 & 0.0548 \\
\hline Medio rural/urbano & $\begin{array}{l}\text { Rural } \\
\text { Urbano }\end{array}$ & $\begin{array}{l}42 / 177(23.7) \\
19 / 169(11.2)\end{array}$ & 9.495 & 0.0021 \\
\hline Nivel socioeconómico & $\begin{array}{l}\text { Bajo } \\
\text { Medio-alto }\end{array}$ & $\begin{array}{l}57 / 258(22.1) \\
4 / 88(4.6)\end{array}$ & 17.256 & $<0.0001$ \\
\hline Genética & $\begin{array}{l}\text { Hereditario } \\
\text { Esporádico }\end{array}$ & $\begin{array}{l}13 / 107(12.1) \\
48 / 239(20.1)\end{array}$ & 3.387 & 0.0657 \\
\hline
\end{tabular}

* P en distribución de $\chi^{2}$ por la razón de verosimilitud.

de medio rural y nivel socioeconómico bajo. La talla baja, además, fue más frecuente en niños procedentes del sur de la república, en especial de los estados de Chiapas, Guerrero y Oaxaca. El resto de las variables no mostró diferencias significativas. 


\section{DISCUSIÓN}

La observación de que los niños con retinoblastoma son de menor talla que la población normal ha sido motivo de controversia y son pocos los estudios que exploran esta característica en forma detallada. ${ }^{22-24}$ Algunas publicaciones han explorado ese dato en niños tratados para investigar el crecimiento en los niños con déficit visual o los que padecen secuelas a largo plazo, pero no se encontraron alteraciones en los valores antropométricos aun cuando no hacen referencia a los valores de esta población antes del tratamiento. Pui y sus colaboradores, en $1987,{ }^{22}$ no encontraron alteraciones en la talla en niños con distintas patologías oncológicas y sugirieron que los informes que señalan talla baja en pacientes oncológicos eran ocasionados por toma inadecuada de las mediciones, series pequeñas, poblaciones de referencia no controladas o por variables que introducían confusión. En un estudio retrospectivo sobre las características clínicas de 52 niños con retinoblastoma, realizado en México por el grupo de Amozorrutia-Alegría, en el Instituto Mexicano del Seguro Social entre 1990 y 1994, no se tomaron en cuenta los valores antropométricos. ${ }^{8}$

En nuestro estudio encontramos que la población de niños con retinoblastoma tenía menores peso y talla que la población general evaluada por la distribución de la puntuación Z con base a los valores del NCHS/CDC; sin embargo, al comparar la proporción de niños de peso y talla bajos con retinoblastoma, con la proporción encontrada por la Encuesta Nacional de Nutrición de 1999, encontramos que con respecto al peso sí hay diferencia pero no con respecto a la talla. Por el contrario, parecería que la proporción de niños con talla baja es aún menor en los niños con retinoblastoma, al menos en el Distrito Federal y en la zona norte del país; aunque en este último grupo hay muy pocos casos para hacer conclusiones definitivas. Los niños con retinoblastoma y peso y talla bajos provienen de poblaciones con nivel socioeconómico bajo, de origen rural y originarios de estados del sur de la república, que son poblaciones con mayor prevalencia de desnutrición.

Concluimos que el peso y la talla bajos que se presentan en una proporción elevada de los niños con retinoblastoma son debidos a factores relacionados con la etapa avanzada de la enfermedad al momento del diagnóstico, la que a su vez se relaciona con el ser originarios de población rural de las regiones más pobres del país.

Una limitante de este estudio es que las medidas de peso y talla fueron tomadas del expediente clínico y no directamente por los investigadores, por lo que puede haber errores de precisión. Sin embargo, estas medidas son las que se requieren en oncología para calcular la dosis de la quimioterapia y se suele ser bastante acucioso al recabar estos datos. Dado que la mayoría de las variables sociales y ambientales están muy relacionadas entre sí, y que no es posible descartar que existan posibles factores genéticos en poblaciones de las diferentes regiones del país, las conclusiones de esta investigación deben ser verificadas con un análisis de las medidas somatométricas de los padres y familiares y establecer grupos de comparación para evaluar estas variables que introducen cierto grado de confusión.

\section{REFERENCIAS}

1. Leal C. Risk of Dying of Retinoblastoma in Mexican Children. Med Pediatr Oncol 2002;38:211-213.

2. Ridaura Sanz C, Rivera Luna R. Cáncer en los niños en México. En: Ruy Pérez Tamayo, El Cáncer en México. Ciudad de México: El Colegio Nacional, 2003; pp. 645-48.

3. Stiller C, Parrin D. Geographic and ethnic variations in the incidence of childhood cancer. Br Med Bull 1996;52:682-703.

4. Smith MA, Gloeckler Ries L.A. Childhood Cancer: Incidence, survival and mortality in Pizzo PA Poplack DG (Eds) Principles and Practice of Pediatric Oncology 4th ed. Philadelphia: Lippincot Williams \& Wilkins, 2002; pp. 1-12. 
5. Linet MS, Ries LAG, SNT. Cancer Surveillance Series Recent Trends in Childhood Cancer Incidence and mortality in the United States. J Natl Cancer Inst 1999;91:1051-57.

6. Rivera Luna R, Leal Leal C, Cardenas Cardós R. A survey of 4076 children with cancer. Certain epidemiological aspects from a single Institution. Bol Hosp Infant Mex 1996;53:598-605.

7. Fajardo Gutiérrez $A$, Mejia Arangure $M$, Gómez Delgado A, Mendoza Sanchez H Epidemiología de las neoplasias malignas en niños residentes del Distrito Federal. Bol Med Hosp Infan Mex 1995;52:507-16.

8. Amozourrutia AV. Epidemiological characteristics of Retinoblastoma in Children Attending the Mexican Social Security Institute in Mexico City, 1990-1994, Paediatric and Perinatal Epidemiology 2002;16:370-374.

9. Ridaura Sanz C. Avances Recientes en la Patologia Neoplásica Pediátrica en Roberto Rivera Luna (editor) Oncología Pediátrica: Conceptos Básicos y Clínicos Ciudad de México: Intersistemas, Capítulo 12. pp. 227-240.

10. Orozco Orozco L, González del Angel A, Saldaña Alvarez Y. Biología molecular del cáncer Gen RB1 Retinoblastoma en Oncología Pediátrica Conceptos básicos y clínicos. Rivera Luna (editor). Ciudad de México: Intersistemas, 2002; pp. 146-147.

11. Mares L, Orozco Orozco L. Estudio Clinicogenético de retinoblastoma. Hosp Infant Mex 1992;49:175-79.

12. Gómez-Martínez R, Leal Leal C, Rivera Luna R. Los aspectos epidemiológicos del retinoblastoma bilateral. Gaceta Médica de México 1995;131:527-32.

13. Schultz K. An Increased Relative Frequency of Retinoblastoma at a Rural Regional Referral Hospital in Miraj, Maharashtra, India. Cancer 1993;72:282- 286.

14. Kingston J, Clark J, Santos H, Jones D, et al , et al, Failure to Thrive Leading to Early Detection of Retinoblastoma. Pediatric Hematology and Oncolgy 1990;7:199-203.
15. Mitter D, Ullmann R, Muradyan A, Klein-Hitpa L, Kanber1 D, Ounap K, Kaulisch M and Lohmann D. Genotype-phenotype correlation in patients with retinoblastoma and interstitial 13q deletions. European Journal of Human Genetics 2011;19:947-958.

16. Saw SM. Incidence and Survival characteristics of Retinoblastoma in Singapore from 1968 to 1995, J Pediatr Ophthalmol Strabismus 2000;37:87-93.

17. US Department of Health and Human Service, Centers For Disease Control and Prevention, National Center for Health Statistics, National Health and Nutrition Examination Survey, Clinical Growth Charts 2001.

18. De Onis $\mathrm{M}$. Measuring nutritional status in relation to mortality, Bulletin of the WHO 2000;78(10):1271-74.

19. WHO, Physical Status: The use and interpretation of anthropometry. Report of a WHO Expert Committee. WHO Technical Report Series No. 845. Geneva, 1995.

20. Rivera Domarco J, Shamah Levy T, Villalpando Hernandez S, Gonzalez de Cosio T, Hernandez Prado B, Sepúlveda J, Encuesta Nacional de Nutrición, 1999. Estado nutricio de niños y mujeres en México Cuernavaca Morelos, México, Instituto Nacional de Salud Pública 2001.

21. Fuente: Archivo Clínico Instituto Nacional de Pediatria

22. Pui CH, Dodge RK, George SL, Green AA. Height at diagnosis of malignancies. Arch Dis Child 1987;62(5):495-9.

23. Peek AML, Schouten-van Meeteren AY, Growth parameters in children with retinoblastoma. Acta Paediatr 1999;88:181-85.

24. Ross G, Lipper EG, Abramson D, Preiser L. The Development of Young Children with Retinoblastoma. Arch Pediatr Adolesc Med 2001;155:80-83. 